\title{
Effect of Geometry Design on Mixing Performance of Newtonian Fluids using Helical Overlapped Mixer Elements in Kenics Static Mixer
}

\author{
A. Talhaoui ${ }^{1 \dagger}$, B. Draoui ${ }^{2}$ and A. Youcefi ${ }^{3}$ \\ ${ }^{I}$ Department of Mechanical Engineering, University of Sciences and Technology USTO-MB, PB 1505 El \\ Mnaouer 31000 Oran, Algeria \\ ${ }^{2}$ Laboratory of Energy of Dry-Zones (ENERGARID), Faculty of Sciences and Technology, University of \\ Bechar, BP 417 Bechar, Algeria \\ ${ }^{3}$ Aeronautics Laboratory and Propulsion Systems, USTO-MB,PB 1505 El Mnaouer 31000 Oran, Algeria \\ †Corresponding Author Email: talhaouiahmed01@gmail.com
}

(Received December 20, 2020; accepted May 3, 2021)

\begin{abstract}
The laminar flow pattern and mixing behavior of incompressible Newtonian fluids in different modified mixer configurations were numerically investigated using Computational Fluid Dynamics (CFD) simulations in the range of $\mathrm{Re}=0.15-100$. The governing equations were solved by ANSYS Fluent 14 using the second-order finite volume method (FVM) and the SIMPLE algorithm scheme. The computational model is assessed by comparing the predicted pressure drop results to empirical correlations in the literature. The effects of incorporated helical overlapped mixer elements and the diameter aspect ratio (C) on the mixing efficiency for different mixer geometries were examined and evaluated by characteristics measures of Intensity of Segregation (IOS), pressure drop, extensional efficiency, and G-factor. The performance of new modified mixers is evaluated via comparison with the standard industrial Kenics static mixer. The static mixers with modified internal geometry achieved fast mixing and better mixing quality than the Kenics mixer. Besides, an increase in diameter aspect ratio $\mathrm{C}$ benefited from a decrease in pressure drop within the static. The modified mixer: $\mathrm{C}=1.5$ was found to have the highest mixing efficiency, concerning short mixing length with marginally higher pressure drop than the other mixers. In contrast, the mixer: $\mathrm{C}=2$ is the most efficient based on low pressure drop and energy requirement with slightly greater mixing length.
\end{abstract}

Keywords: Mixing; Laminar Flow; Static mixer; Overlapped mixer; Mixing performance; Mixer efficiency.

\section{NOMENCLATURE}

$\begin{array}{ll}A R & \text { length to diameter aspect ratio } \\ C & \text { diameter aspect ratio } \\ D & \text { diameter of the mixer } \\ d & \text { diameter of overlapped mixer elements } \\ D_{A B} & \text { molecular diffusion coefficient } \\ D_{h} & \text { hydraulic diameter } \\ F & \text { source term force } \\ h & \text { concentration of tracer } \\ \bar{h} & \text { mean concentration } \\ i & \text { data point } \\ I O S & \text { intensity of Segregation } \\ L & \text { length of the mixer } \\ L^{*} & \text { dimensionless length of the mixer } \\ l_{b} & \text { length of the mixing blade }\end{array}$

$\begin{array}{ll}\Delta P_{m i x} & \text { pressure drop in the mixer } \\ R & \text { radius of the mixer } \\ R^{*} & \text { dimensionless radius of the mixer } \\ R_{e} & \text { Reynolds number } \\ R E & \text { Relative Error } \\ t & \text { time } \\ t_{e} & \text { thickness of the blade } \\ U & \text { velocity vector } \\ U_{m} & \text { mean velocity } \\ U^{*} & \text { dimensionless velocity } \\ x, y, z & \text { Cartesian coordinate axes } \\ Y_{i} & \text { local mass fraction of species } \\ Z_{f} & \text { Z factor } \\ Z^{*} & \text { dimensionless axial distance }\end{array}$




$\begin{array}{ll}l_{e} & \text { length of the overlapped blade } \\ l_{\text {in }} & \text { entrance length prior } 1 \text { st mixer element } \\ l_{\text {out }} & \text { outlet length after } 6 \text { th mixer element } \\ M R E & \text { Mean Relative Error } \\ N & \text { number of data points } \\ P & \text { pressure } \\ \Delta P_{\text {emp }} & \text { pressure drop in empty pipe }\end{array}$

\section{INTRODUCTION}

Static mixers or so-called in-line reactors have become popular mixing devices in a broad scope of applications in the industrial process; polymer melts, pharmaceutical formulations, petroleum, paint, and food processing (Asprion et al. 2011, Caserta et al. 2013, Tian and Barigou 2015). Static mixers are utilized in complex heat and mass transfer processes, such as dispersing, contacting, blending, reactions, and heat exchange. They are employed as an alternative mixing device of conventional agitated vessels since similar or higher mixing performance can be obtained at low cost and in small space. Some advantages of static mixers over dynamically agitated vessels are the motionless parts, then less power consumption, where the required energy is directly provided in line by the pump that drives the flow through the mixer, and no additional energy is required. The static mixers are selected for their fast mixing process, high mixing quality, narrow residence time distributions, self-cleaning, and low maintenance requirements. More advantages of static mixers are well described in the literature Hobbs and Muzzio (1997), Thakur et al. (2003), Regner et al. (2006), Ghanem et al. (2014).

The mixing in static mixers is attained by the movement of the fluid streams getting twisted, chopped, rotated, and recombined as they flow through the internal geometry of static elements. The resulting pressure drop in the static mixer is converted into mechanical energy Song and Han (2005). Thus, high mixing performance and minimum pressure drop are the essential criteria for selecting an ideal static mixer. However, the pressure drop is not a standard measure of the mixing efficiency. Still, it is an essential parameter at the design stage because it directly affects the energy that governs the mixing cost. In general, the blade shape imparts the momentum spin to the fluids that change direction with each succeeding element by following the curvature of the blade shape. Fluids constantly move outward from the pipe center to the wall pipe and back again, with the interface between elements in the active zone. As a result, the pressure drop is relatively higher in the compact mixer of a high volume of mixer elements (low void fraction). The importance of structural properties of blade shape design and manufacturing, in practice, may highly affect the pressure drop in the mixer Heniche et al. (2005). Other designs of static element geometry were developed ranging from open to

$\begin{array}{ll}\gamma & \text { deformation tensor } \\ \lambda & \text { extensional efficiency } \\ \mu & \text { dynamic viscosity of } \\ \omega & \text { vorticity tensor } \\ \rho & \text { fluid density } \\ \sigma_{h} & \text { standard deviation of concentration }\end{array}$

close, depends on the conditions and the requirements of the mixing system Ghanem et al. (2014), Meng et al. (2016b), Soman and Madhuranthakam (2017), Haddadi et al. (2020).

The Kenics Static Mixer (KSM) is a versatile mixer largely employed in mixing operations. It increases the stretching, splitting, folding, reorientation of fluid streams. The set of inserts generates a high interfacial area for fluids being mixed, consequently increases the mixedness degree (Rafiee et al. 2013, Haddadi et al. 2020). It is highly recommended to mix high viscous materials in laminar flow when the turbulent flow is impractical or limited due to high pressure and a considerable amount of required power. The standard design of the industrial Kenics mixer is composed of a cylindrical channel fitted with a set of helical blades (Static Mixing Elements). The helical blades are alternatively twisted clockwise and counter-clockwise with a twist angle of $180^{\circ}$. Each mixing element is inserted and aligned axially along the channel, so the trailing edge of each blade is perpendicular to the leading edge of the succeeding one ( Lisboa et al. 2010, Thakur et al. 2003, Murasiewicz and Jaworski 2009, Meng et al. 2015). They direct the flow in the radial direction into the mixer walls and back to the center. Concurrently, the flow division patterns are produced and grow exponentially as the flow passes through each blade (Thakur et al. 2003, Vilar et al. 2017, Mahammedi et al. 2017).

For an appropriate selection of a static mixer for particular mixing applications, the characteristics of the mixing system, such as; flow conditions, mixing efficiency, and internal geometrical characteristics of the static mixer have to be considered and thoroughly understood. At the designing stage, the experimental investigations of the mixing degree and the performance of different static mixers require a repeated set of experiments, which turn it a costly expensive, and time-consuming task, particularly for three-dimensional flows in complex geometries. The complex geometries and flow complexity of most mixers in industrial processes make analytical solutions for the velocity field within these mixers not practical Hobbs and Muzzio (1997). To investigate such flows in complex Geometries of practical interest, the Computational Fluid Dynamics (CFD) analysis is an alternative tool for characterizing the mixing performance in complicated mixer geometries. It is applied in a broad range of flow conditions and different types of fluids as well, even when the developed flows are 
fully three dimensional (Jovanovi et al. 2014, Ramsay et al. 2016, Jegatheeswaran et al. 2017, Stec and Synowiec 2019, Haddadi et al. 2020, Soman and Madhuranthakam 2017). Several studies have numerically and experimentally been carried out to characterize the mixing behavior in the Kenics mixer in recent years. Different measures and criteria are used to examine the mixing performance in the static mixers based on numerical results. The commonly employed measures are pressure drop, residence time distribution, stretching rate, Lyapunov exponent, striation thinning, the intensity of segregation, extensional efficiency, and variation coefficient. Hobbs and Muzzio (1997)conducted a numerical study of the properties and characteristics of chaotic mixing in Kenics mixer for flows of low Reynolds number. It was found that the average stretching rate in the chaotic flow grew exponentially when the number of flow periods is increased by increasing the number of mixing elements inside the static mixer. Saatdjian et al. (2012) also investigated the chaotic advection and mixing performance within a modified geometry by symmetry breaking of the standard kenics static mixer using different locations of injection. The mixing efficiency of the new modified geometry is compared with the standard configuration of the Kenics mixer. It was reported that the influence of injection location on the mixing performance is trivial when the mixing elements within the mixer are sufficiently high.

Additionally, the mixing is slightly improved in modified geometry. It improves the homogeneity distribution of stretching rate and the extensional efficiency in a wide range of Reynolds numbers due to chaotic flow. The impact of blades twist angle, and the direction of their arrangement inside the mixer on the mixing performance in the Kenics static mixer has also been studied using variation coefficient and dynamical systems techniques Hobbs and Muzzio (1998). They showed that a Kenics mixer with $120^{\circ}$ twist angle provides $44 \%$ more efficient energy than the Kenics mixer(KSM). Also, the mixing quality of static mixer with standard blades layout Right-Left (R-L) is superior upon the mixer with the same twist direction Right-Right (R-R) blades configuration because of unmixed regions with no mass transfer with the entire flow. In the same context, Galaktionov et al. (2003) characterized the mixing performance in the Kenics mixer in a broad range of twist angles with an increment of $5^{\circ}$ add to different twist direction Right-Left (R-L) and Right-Right (R$\mathrm{R})$ layouts. They used the mapping technique, combined with the discrete intensity of segregation, to assess mixing quality. It was found that the Kenics mixer with blades of twist angle in the same direction is inefficient to homogenize components because of the presence of segregated islands. Nevertheless, it was also found that not all configurations have dead zones. However, the global mixing is achievable at an optimal twist angle equal to $140^{\circ}$ with an (R-L) layout.

Regner et al. (2006) performed a comparative study of Kenics static mixer and Lightnin series 45 mixer. They showed that the intensity of vortices in KSM is lower than the Lightnin mixer due to the curved shape and edges in the middle of the blades in the Lightnin mixer. Additionally, the formation of vortexes increases the required energy due to high pressure drop and increases the rate of striation thinning. At high flow rates, segregated streaks pop up that reduce the inter-material area and lower the mixing. For high viscous fluids, the mixing performance and chaotic laminar flow in the Lightnin mixer were computationally investigated and compared with the standard Kenics static mixer at $\mathrm{Re}=0.1-100$ using different aspect ratios, as well as a various number of mixing elements Meng et al. (2016a). They reported that the friction coefficient is decreased in the Lightnin mixer with the increase of Reynolds number, and Lightning mixer exhibited better distribution of mixing over Kenics static mixer due to high energy input. Besides, they suggested a new factor $\eta$ for evaluating the performance of micro-mixing depends on the tradeoff between friction coefficient and the increase of the stretching rate. Moreover, several researches have been performed using CFD tools to characterize the mixing in Kenics static mixer Byrde and Sawley (1999), Bakker et al. (2000), Rauline et al. (2000), Rahmani et al. (2008), Zhang et al. (2015).

Numerous works have investigated the performance of mixing in Kenics and novel static mixers. Some computational studies analyzed mixing in innovative static mixers or design modifications upon the existing mixers. Haddadi et al. (2020) numerically explored the mixing behavior of two miscible fluids in a new innovative static mixer for Reynolds numbers range 20 to 160 . They compared the mixing performance in the novel mixer with the Kenics, Komax, and SMX static mixers. It was reported that the innovated mixer exhibits higher mixing performance than the other comparative mixers, where the $\mathrm{Z}$-factor is decreased about $45 \%$ compared with the SMX mixer in the same operating conditions. Also, the new mixer provides high extensional efficiency revealing the presence of both high dispersive and distributive mixing. Meng et al. (2014) computationally examined the mixing performance of multi-twisted leaves innovative static mixers of high viscous fluid in the range of $\mathrm{Re}=0.1-150$. Three different modified geometries were investigated; the Kenics static mixers with double twisted leaves (DKSM), three twisted leaves (TKSM), and four twisted leaves (FKSM).

The extensional efficiency, G-values, particle distributions, and stretching rate were used to assess the mixing performance in the novel mixers. Their findings stated that TKSM and FKSM reached fast chaotic mixing due to the compactness and curvature of blades that potentially improve the dispersive mixing. In contrast, larger unmixed segregated zones were obtained with (DKSM). There are small regions of great stretching; higher mixing efficiency is obtained by TKSM and FKSM than the DKSM and KSM. In the same context of internal geometry modification in the static mixer, Meng et al. (2016b) used CFD tools to investigate the performance of perforated blades of a modified Kenics static mixer in the turbulent flow. The impact of holes, size, spacing and the number of mixing elements on the 
overall performance of heat transfer were analyzed. It was shown that the best performance achieved in terms of friction losses and heat exchanger with perforated blades of $(\mathrm{d} / \mathrm{W}=0.3$ and $\mathrm{s} / \mathrm{W}=0.6)$ compared to the standard Kenics mixer.

As can be seen from the past investigations and studies, new static mixer designs are being innovated and improved, and new applications are discovered as well. Despite the availability of several works that investigated the impact of the geometry modifications on mixing performance in static mixers, but they remain insufficient to understand the mixing behavior in the static mixers thoroughly. Considering the studies mentioned, the impact of the geometrical parameters of overlapped helical mixer elements in the Kenics static mixer has not been studied yet. Thus, this study aims to investigate the hydrodynamics of flow and mixing behavior of a mixture of two miscible Newtonian fluids in a new modified static mixer with overlapped helical blades in a range of Reynolds number 0.015-100. The effects of incorporated overlapped helical mixer elements and diameter aspect ratios between the main and overlapped mixer on the mixing performance were examined using CFD tools. Different criteria have been employed to assess the performance of the different mixer geometries, including the pressure drop, the extensional efficiency and G-factor, and the Intensity of Segregation (IOS) of tracer particle distributions. The CFD data are validated via comparison of the predicted computational results of pressure drop to the existing empirical correlations in the literature.

\section{NuMERICAL Methodology}

\subsection{Governing equations}

For three-dimensional and incompressible flows. The governing equations of continuity, momentum and species conservation equations for laminar flow without reactions are given as follows:

$\frac{\partial \rho}{\partial t}+\nabla \cdot(\rho U)=0$

$\rho \frac{\partial U}{\partial t}+U \cdot \nabla(\rho U)=-\nabla P+\mu \nabla^{2} U+F$

$\frac{\partial}{\partial t}\left(\rho Y_{i}\right)+U . \nabla\left(\rho Y_{i}\right)=\rho D_{A B} \nabla^{2} Y_{i}$

Where $\rho$ denotes the fluid density, $U$ stands for velocity vector, $\mu$ is the dynamic viscosity of the fluid, $t$ is the time, $P$ is the pressure, $Y_{i}$ is the mass fraction, $D_{A B}$ is the molecular diffusivity, and $F$ stands for source term including volumetric forces.

The governing equations mentioned above were discretized using the finite volume approach. The second implicit scheme and pressure-based solver were employed for the flow simulations in all static mixers. The SIMPLE algorithm was used to handle the coupling pressure-velocity, and computations of momentum, pressure, and species transport were carried out using second-order upwind schemes. Gradients are required for computing the velocity derivatives, diffusion, and convective terms in the flow. The computations are performed using ANSYS FLUENT 14.0 software.

The convergence limit for residuals of the conservation equations is below $10^{-6}$. Accordingly, the employed incompressible fluid in all simulation is water with following physical properties, viscosity $\left(\mu=0.001 N . s / \mathrm{m}^{2}\right)$, density $\left(\rho=1000 \mathrm{Kg} / \mathrm{m}^{3}\right)$. The mass diffusion coefficient $D_{A B}=1.5 e^{-9} \mathrm{~m}^{2} / \mathrm{s}$ which is suitable for aqueous solutions. It is worth mentioning that all simulations were run in a steady state.

\subsection{Geometry and Grid}

The industrial design of the Kenics static mixer is used as reference geometry for numerical simulations and further comparisons with the new modified geometries. The standard design of the Kenics static mixer is composed of a cylindrical tube of diameter $\mathrm{D}=10 \mathrm{~mm}$ and a total length of $140 \mathrm{~mm}$, as shown in (Fig. 1-a). The diameter of blades is the same as the internal diameter of the pipe with length $\mathrm{l}_{\mathrm{e}}=15 \mathrm{~mm}$, the length to diameter aspect ratio of each blade is $\mathrm{AR}=\mathrm{L} / \mathrm{D}=1.5$, and thickness $\mathrm{t}_{\mathrm{e}}=1 \mathrm{~mm}$. A tube length of $2.5 \mathrm{D}\left(\mathrm{l}_{\text {in }}=\mathrm{l}_{\text {out }}\right)$ is kept empty ahead and after the mixing blades to ensure sufficient flow length to deter the creation of backflow effect into the domain of simulation. Four models were constructed with various diameter aspect ratios; $\mathrm{C}=\mathrm{D} / \mathrm{d}=1.25,1.5$, and 2; where $\mathrm{d}$ stands for the diameter of the overlapped helical blades, with similar blade length $\mathrm{l}_{\mathrm{b}}=15 \mathrm{mmn}$ as illustrated in (Fig. 1-b). Table 1 displays the corresponding dimensions of different mixers and additional geometrical parameters. With the facilities and technology available nowadays, it became easy to design highly sophisticated mixing elements of different sizes. The 3D printed objects techniques provide high manufacturability convenience for designing new mixers with complex geometry and shapes. Although, scaling up to industrial manufacturing always remains strenuous due to the production scale and process conditions. Each modified mixer has 12 mixing elements (6 mixer elements of the main mixer and six overlapped mixer elements). The overlapped helical blades are inserted in the channel in the opposite twist direction of the main blades, i.e., the main blades are RightLeft (R-L) set, and the overlapped are Left-Right (L$\mathrm{R})$, both with similar twist angle 180 . The various geometrical models of mixers and corresponding grid are constructed and generated using ANSYS 14.0; the unstructured tetrahedral mesh was used for discretization of the computational domain. The tetrahedral grid of the new overlapped mixer is shown in (Fig. 1c). The likewise simulated geometry with the tetrahedral mesh of the computational domain is illustrated in (Fig. 2). To evaluate the mixing efficiency and mixture homogeneity of two fluids with the same amount and enable the second phase, the inlet of each mixer is split into two evenly 


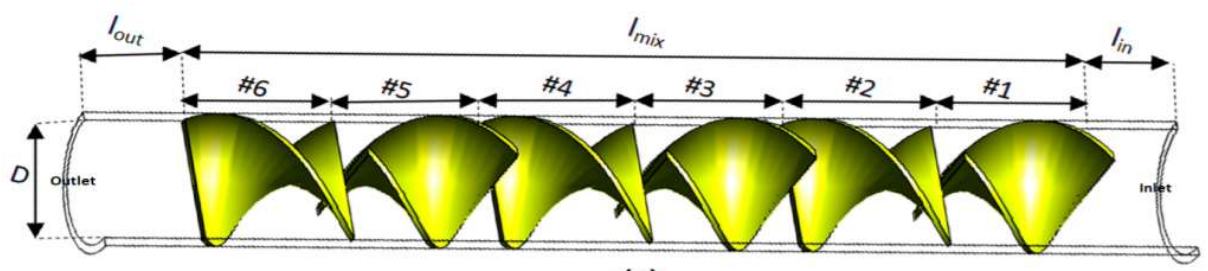

(a)
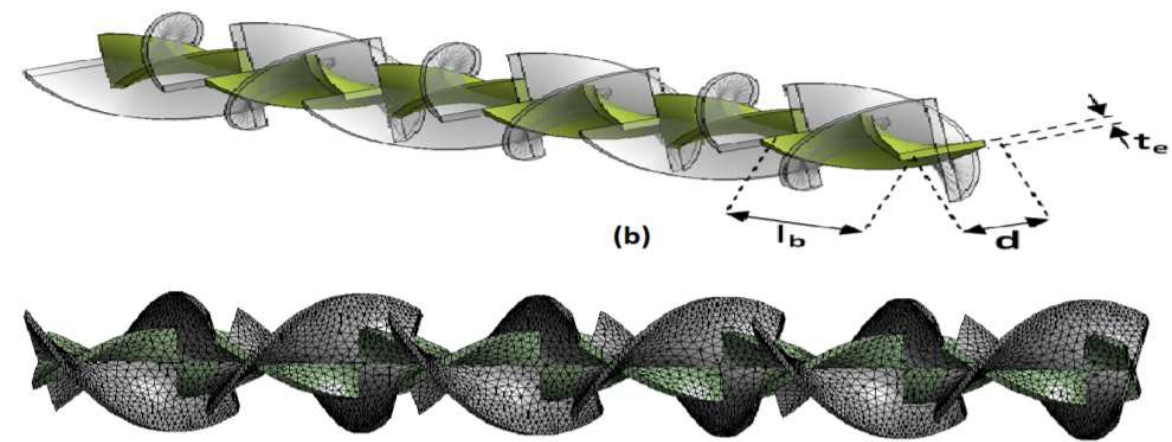

(c)

Fig. 1. (a) Standard Kenics static mixer, (b) overlapped static mixer and (c) tetrahedral grid of overlapped mixer.

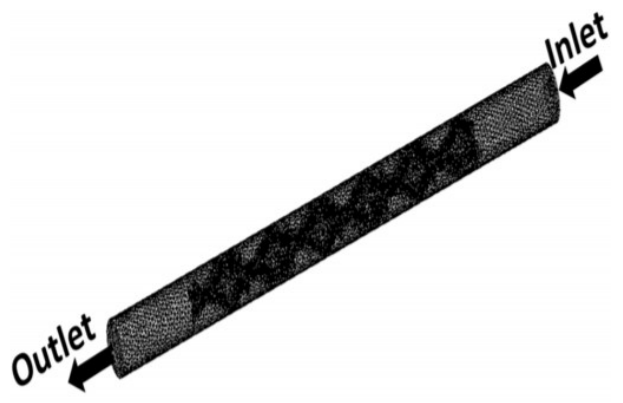

Fig. 2. Unstructured Tetrahedral grid of the flow domain.

semi-circles. The first fluid flows in the mixer through one semi-circle inlet, and the second fluid flows through the second inlet.

\subsection{Boundary conditions}

The Reynolds number (Re) governs the flow conditions in mixing systems. It is defined as:

$R_{e}=\frac{\rho U D_{h}}{\mu}$

Where: $D_{h}$ is the hydraulic diameter. The governing equations were solved numerically using the following boundary conditions: the velocity inlet sets at inlets of the mixer, and pressure outlet is defined at the mixer outlet. No-slip stationary boundary condition was assumed for all solid walls. A fully developed velocity profile was imposed using the User-Defined Function (UDF) with zero pressure gradient at the inlets, and the static pressure at the mixer outlet is Zero. Moreover, the mass fraction values were set to be " 0 " at inlet $\mathrm{A}$ where the primary liquid is flowing in the mixer and "1" at the other inlet $t_{\mathrm{B}}$ for the tracer. It is worthy to note that the tracer has the same physical properties as the primary working liquid.

\subsection{Grid independence solution}

In this part, we present the independent grid solution; thus, different velocity profiles of various grid sizes were compared. Therefore, we define the following non-dimensional variables:

$U^{*}=\frac{U}{U_{m}} ; R^{*}=\frac{2 R}{D} ; Z^{*}=\frac{Z}{L}$

Where: $U_{m}$ denotes the average velocity, $R$ the radius of the mixer, $Z$ the axial distance, and $L$ is the length of the mixer. After checking and editing the mesh, the initial unstructured mesh was generated and exported to the solver. The grid size was increased from $0.25 \mathrm{~mm}$ to $0.75 \mathrm{~mm}$ at $\mathrm{Re}=100$ with the maximum equisized skew value of 0.78 . The grid sensitivity test of the dimensionless velocity profiles corresponding to different grid sizes of 0.35 , $0.4,0.45,0.5$, and $0.6 \mathrm{~mm}$ at the mixer outlet is shown in Fig. 3 . The velocity profile decreased by $1.2 \%$ as the grid size increased from $0.35 \mathrm{~mm}$ to $0.4 \mathrm{~mm}$. While increasing the grid size to $0.45 \mathrm{~mm}$ and $0.5 \mathrm{~mm}$, the velocity profile altered with a decrease of $0.37 \%$ and $3.5 \%$ respectively; meanwhile, the grid size of $0.6 \mathrm{~mm}$ gave a sharp increase of the velocity of $8.2 \%$. Therefore, we have chosen $0.4 \mathrm{~mm}$ as grid size for all simulations because of the high computational accuracy and reduced simulation time.

\subsection{Model validation}

In this section, the reliability and accuracy of the CFD model employed in this work are validated. The pressure is an important parameter that is very responsive to the flow perturbations comparing to 
Table 1 Specification of geometries.

\begin{tabular}{|l|c|c|}
\hline & Standard Kenics Mixer & New modified Mixer \\
\hline Length, L $(\mathrm{mm})$ & 140 & 140 \\
\hline Diameter, D $(\mathrm{mm})$ & 10 & 10 \\
\hline $\mathrm{lb}(\mathrm{mm})$ & 15 & 15 \\
\hline Linlout $(\mathrm{mm})$ & 25 & 25 \\
\hline Length to diameter aspect ratio AR $(-)$ & 1.5 & 1.5 \\
\hline Twist angle, (degree) & 180 & 180 \\
\hline Diameter aspect ratio C $(-)$ & - & $1.25,1.5$ and 2 \\
\hline
\end{tabular}

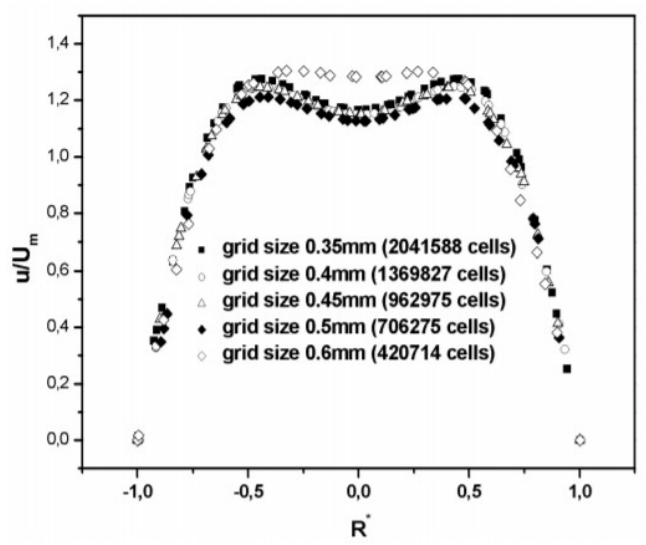

Fig. 3. Grid independency test; Dimensionless velocity profile at the outlet for different grid sizes, $\mathbf{R e}=100$.

concentration and velocity. Accordingly, if the pressure results are satisfactory, the velocity field is likely to be well described Rauline et al. (1998). Therefore, the validation has been carried out via comparison of computed values of pressure drop within mixers with similar geometrical parameters to the pressure drop of the empirical correlations available in the literature. The majority of the available correlations for pressure drop are expressed in $Z$ factor $Z_{f}$. It is defined as the pressure drop ratio through the static mixer to the pressure drop in an empty pipe. The $\mathrm{Z}$ factor is computed as follows:

$Z_{f}=\frac{\Delta P_{m i x}}{\Delta P_{e m p}}$

Where $\Delta P_{\text {mix }}$ denotes the pressure drop in the static mixer and $\Delta P_{e m p}$ denotes the pressure drop of an empty pipe with equivalent length. The pressure loss in empty pipe is calculated via stokes equation:

$\Delta P_{\text {emp }}=\frac{32 L}{\operatorname{Re}} \frac{\rho U^{2}}{D}$

Grace (1971) has proposed a correlation of pressure drop in Kenics mixer for Reynolds values $R e<1000$ :

$Z_{f}=4.86+0.6804 \sqrt{R e}$
In the same context Wilkinson and Cliff (1977) have also posited another correlation for Reynolds values $\operatorname{Re}<50$ :

$Z_{f}=7.19+0.03125 R e$

The accuracy of CFD results is assessed using the mean relative error (MRE) and Relative error (RE).

$$
\begin{aligned}
& M R E=\frac{1}{N} \sum_{i=1}^{N} R E_{i} \\
& R E_{i}=\frac{\mid \text { Numerical }_{i}-\text { Experimental }_{i} \mid}{\mid \text { Experimental }_{i} \mid}
\end{aligned}
$$

Where: i refers to a data point, and $\mathrm{N}$ stands for the number of data points in the system. The CFD values of pressure drop were compared to the two empirical correlations given above, as illustrated in Fig.4. It is clearly shown in Fig. 4, There is a satisfactory agreement with empirical correlations with an overall discrepancy of $2.02 \%$ for Grace (1971) and $2.93 \%$ for Wilkinson and Cliff (1977) correlation respectively, particularly for the Reynolds numbers less than $50 \quad(R e<50)$. Whereas, for Reynolds numbers greater than $50(R e<50)$, there is a slight variation between the CFD results and the Wilkinson and Cliff (1977) experimental correlation because the latter is conceived for Reynolds number not exceeding the value $\mathrm{Re}=50$.

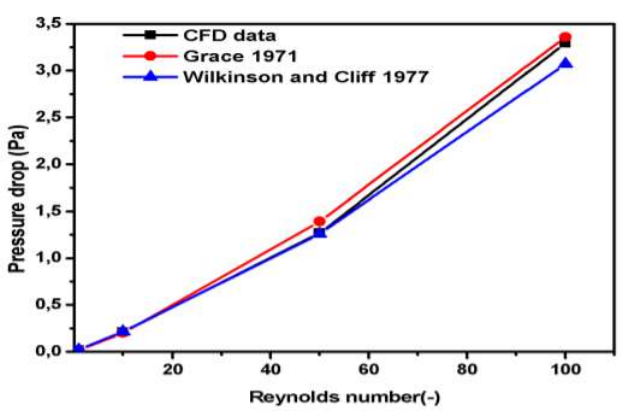

Fig. 4. Computed pressure drop Vs Empirical correlations for various Reynolds numbers. 

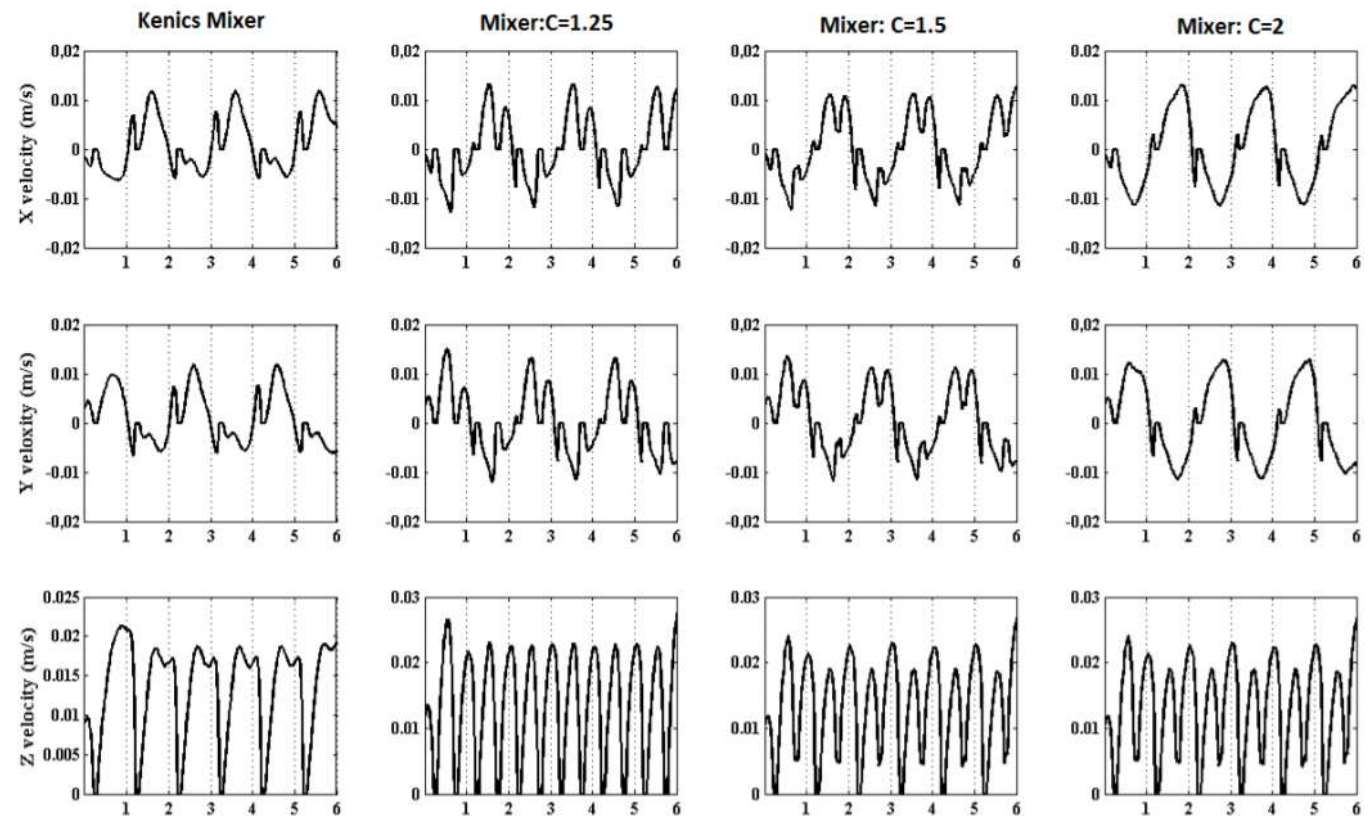

Fig. 5. Three Dimensional velocity profiles along the axial dimensionless position at $\operatorname{Re}=100$. (Rows: 1-x velocity, 2-y velocity and 3-z velocity, Columns:1-Kenics mixer, 2-Mixer): $(C=1.25$, 2-Mixer: $C=1.5,2$-Mixer: $C=2$. $)$.

For instance, at $\mathrm{Re}=100$, the Relative error $(\mathrm{RE})$ values of the computational results and correlations of both Grace (1971) and Wilkinson and Cliff (1977) are approximately $1.92 \%$ and $4.02 \%$ respectively, which are in perfect quantitative agreement. Hence the comparison of numerical results and empirical correlations is exceptional, revealing the reliability of the CFD model used in this work and its ability to predict the mixing behavior in the modified static mixers with satisfactory accuracy.

\section{RESULTS AND DISCUSSION}

\subsection{Velocity profiles}

For analysis of velocity fields, three-dimensional velocities in $\mathrm{x}, \mathrm{y}$, and $\mathrm{z}$ directions are investigated in the axial line parallel to the mixer centerline in the (z-direction) at radial positions $(\mathrm{x}=\mathrm{R} / 2, \mathrm{y}=\mathrm{R} / 2)$ and $\mathrm{Re}=100$. The velocity profiles of the different mixers as a function of dimensionless axial position are illustrated in Fig. 5. It is clearly shown that all mixers exhibit a certain periodicity of velocity profiles due to the spatial periodicity of the physical geometry of mixers. For all mixers, the axial velocity in the $\mathrm{z}$ direction is greater than 0 , and the number of peaks of velocity fluctuations increased for the modified mixers as a result of increasing the number of mixing elements in the mixer (additional overlapped mixer elements). The axial velocity in the $z$-direction is unevenly fluctuated because of the difference in diameter of inserted elements, causing flow instabilities in the mixer and highly contribute to the axial mixing. In addition, the radial velocities in the $\mathrm{x}$ and $\mathrm{y}$ directions are sharply increased as the number of mixer elements increased. Also, the radial flow instability is continuously improved because of the secondary flow in cross section along the mixer, which notably improves the mass diffusion in radial directions.

For more elucidation, the velocity contours at different locations in the mixers for the Reynolds number $\mathrm{Re}=50$ are illustrated in Fig. 6. Since the flow is periodically repeated, the cross sections subjected to analysis were chosen in the third mixing element at different dimensionless distances $0.39<Z^{*}<0.5$. Simultaneously, the velocity streamlines are depicted at the end of trailing edge of the third blade and the beginning of the leading edge of the fourth blade $0.495<Z^{*}<0.51$ as shown in Fig. 7. It is remarked that higher velocities are obtained near the blades for all types of mixers, particularly near the edges of the overlapped blades due to compactness and flow resistance resulting from extra blades and sharp change in geometry. As the fluids flow forward through the mixing elements, fluid streams get split and recombined again, forming new zones with different velocities, mainly in the intersection zone of two successive blades. Because of the non-uniform velocity combined with approximately uniform pressure gradient, several spanwise vortexes are produced in the center near the blades at the junction zone.

Furthermore, Fig. 7 shows the formation of vortexes at end of the trailing edge of the third mixing element, precisely at a distance of $0.4 \mathrm{~mm}$ $\left(Z^{*}=0.497\right)$ upstream the tip of the third blade. The vortices get increased in size near the body of blades until they reach the maximum at the intersection region (the center of vortices is marked with black 
A. Talhaoui et al. / JAFM, Vol. 14, No. 6, pp. 1643-1656, 2021.

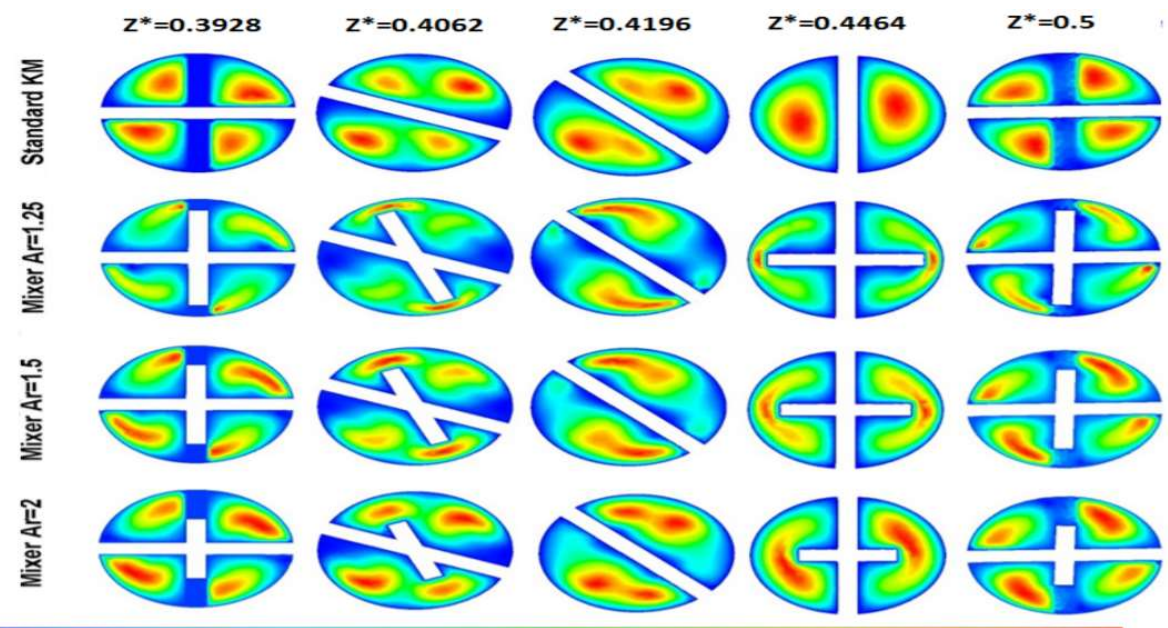

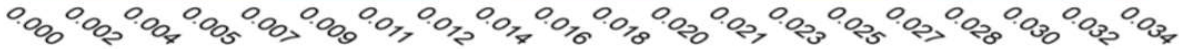

Fig. 6. Velocity contours of different mixer geometries at various locations along the 3rd mixer element at $\mathrm{Re}=\mathbf{5 0}$.

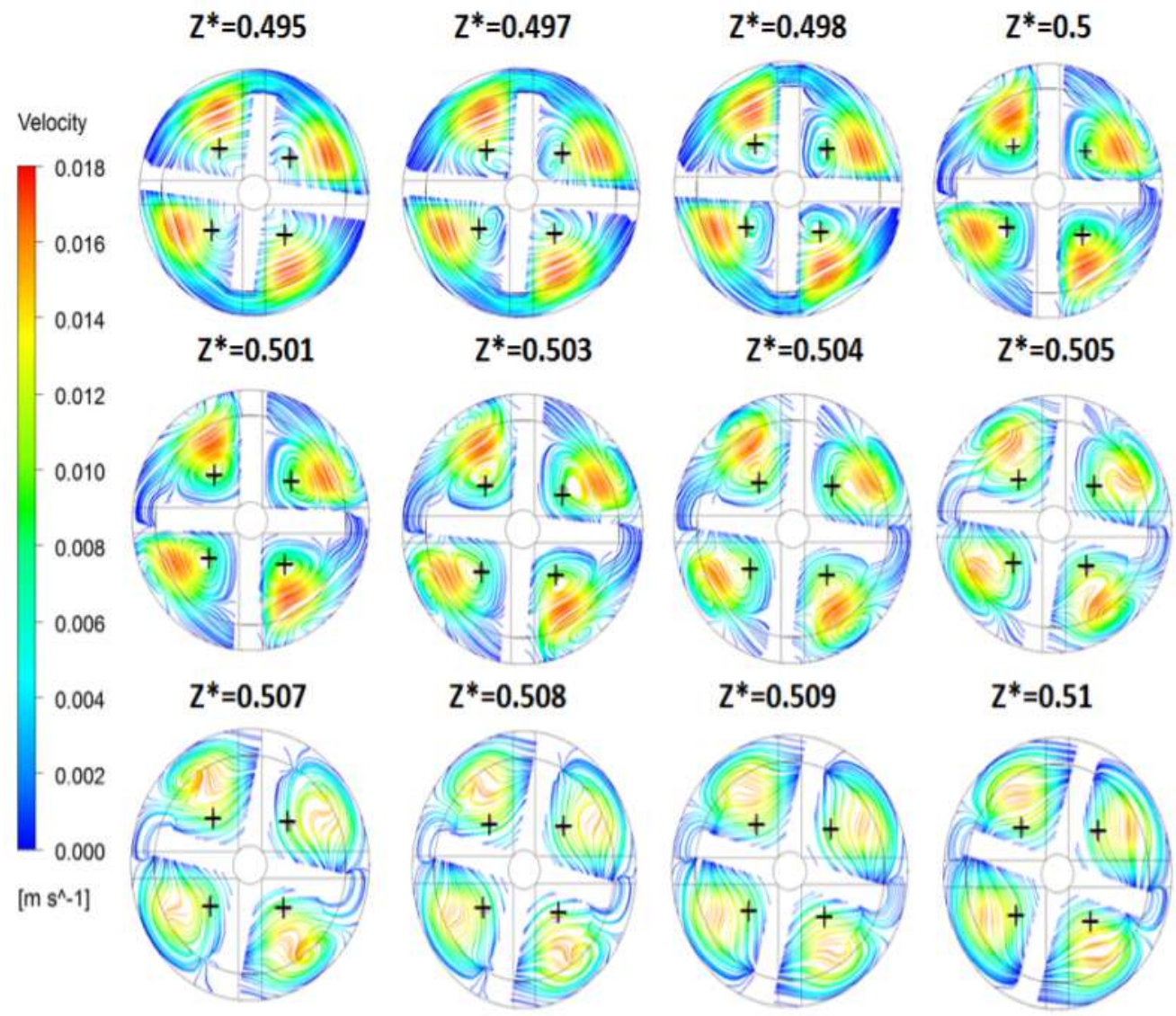

Fig. 7. Velocity streamlines at $3 \mathbf{r d}$ and 4 th mixer elements at $\mathbf{R e}=\mathbf{5 0}$.

cross symbol at the intersection zone). As the flow moves forward through the leading edge of the fourth blade, the vortices begin breaking up into small sizes and then convected away towards the pipe wall due to the curved shape and combined set of overlapped blades. At a distance of $1.4 \mathrm{~mm} \quad\left(Z^{*}=0.509\right)$ downstream the fourth blade, the vortices completely vanish. This is attributed to the continuous flow split and domination of radial mixing, then, the flow aligns with the curvature of the mixing elements. 

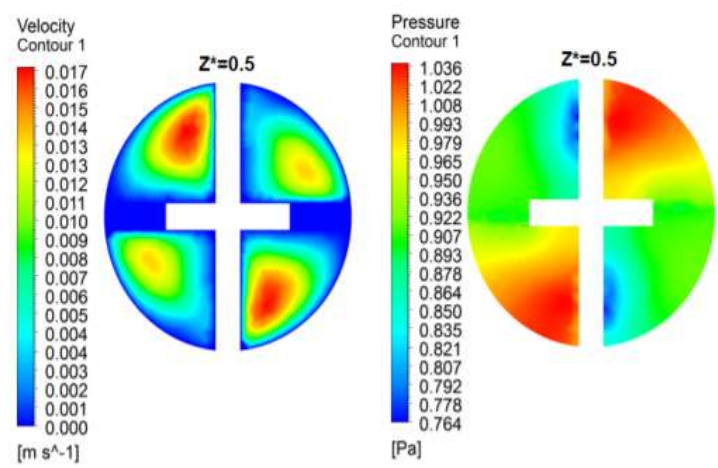

Fig. 8. Velocity and pressure contours at the intersection zone between the $3 \mathrm{rd}^{-4^{\text {th }}}$ mixing blades at $\mathbf{R e}=\mathbf{5 0}$.

This flow pattern has also occurred in the Kenics mixer with moderate intensity comparing to the new mixers. The effect of vortex on the velocity and pressure drop is pronounced in Fig. 8. It clearly shows the combination of four zones of high velocities together at the intersection region, among which two zones have lower pressure that indicating the clockwise or anti-clockwise rotation directions of the vortices. A low pressure is found at the trailing edge of the succeeding blade, and high pressure is associated with high velocity core convected by the flow and strike the blade. Interestingly, the incorporation of overlapped mixer elements, their perpendicular arrangement inside the mixer (i.e., the arrangement with angle $90^{\circ}$ ), and the chaotic advection of fluid particles considerably enhance the mixing performance.

\subsection{Quantitative Mixing analysis}

\subsubsection{Extensional efficiency}

The assessment of Elongational flows leads to recognizing the behavior of mixing that occurs in the static mixer. For this aim, we define the extensional efficiency with the parameter $\$ \backslash$ lambda $\$$. It is used as a characteristic parameter to quantify the efficiency of the Rotational and Elongational flow components Manas-Zloczower (1994). The parameter $\lambda$ can have values between 0 for pure rotational flow and 1 for pure elongational flow, whereas the value of 0.5 stands for simple share flow. The extensional efficiency is essential in evaluating the flow pattern and dispersive mixing efficiency. The greater the parameter $\$$ lambda $\$$, the more the dispersive mixing occurs. It is expressed as follows:

$l a m b d a=\frac{|\gamma|}{|\gamma|+|\omega|}$

where $|\gamma|$ and $|\omega|$ are the magnitudes of rate of deformation and vorticity tensors respectively, that are given as:

$$
\begin{aligned}
& |\gamma|=\frac{1}{2}\left(\nabla V+\nabla V^{T}\right) \\
& |\omega|=\frac{1}{2}\left(\nabla V-\nabla V^{T}\right)
\end{aligned}
$$

$\nabla V:$ is the velocity gradient. Figure 9 displays the extensional efficiency $\lambda$ for different mixers at an axial line parallel to the mixer centerline ( $\mathrm{z}$ direction) at radial positions $(x=R / 2 y=R / 2)$. The high extensional efficiency values are obtained at the middle of the mixing element for the Kenics mixer due to their helical shape. In the intersection zone of mixing elements, the value of $\$$ lambda $\$$ is around 0.5 , explaining the simple shear flow. The latter, combined with the helical shape of the blades, contribute to the dispersive mixing.

On the other hand, for the modified mixers in Fig. 9(b-d), the maximum values of extensional efficiency are obtained at the transition zones of two consecutive mixing elements and the middle of each mixing element. High values indicate the dispersive mixing at blade edges and distributive one along the helix body of the mixing element. The extensional efficiency values reveal a complex flow pattern due to the secondary radial flow generated by the overlapping blades. The elongational flow is predicted at the intersection of two elements, indicating the vital role of the transition part in the stretching of materials. The abrupt change of geometry highly contributes to dispersive mixing. The overlapped mixer elements generate the effect of secondary radial flow. It is clearly shown in Fig.9 that modified mixers are more efficient than the Kenics mixer. A high effective stretching rate is obtained by the mixer with $\mathrm{C}=2, \mathrm{C}=1.25$, and $\mathrm{C}=1.5$, respectively, explaining the intensity of the dispersive mixing in each mixer. As remarked, the values of extensional efficiency are not proportional to the diameter aspect ratio. Based on the analysis given above, it is concluded that high extensional efficiency is not sufficient for the decision of an overall efficient mixer as a consequence of the presence of segregated regions.

\subsubsection{G-Factor}

The G-factor is an indicating parameter of the power as a function of unit volume. It was initially developed in 1943, and it determines the mixer performance of stirred tanks Camp (1943). Recently, the G-factor has gained more interest as a measurement of characterization of the mixing 

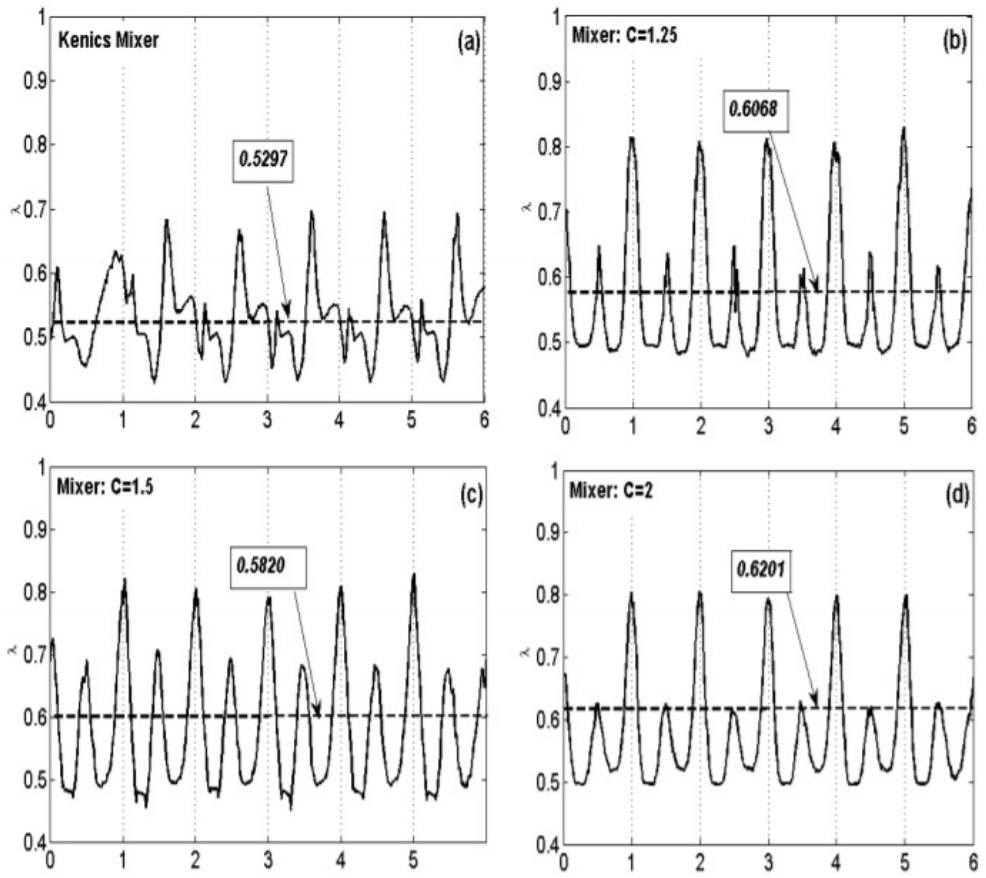

Fig. 9. Extensional efficiency of different mixer geometries at $\mathrm{Re}=\mathbf{0 . 1 5}$.(a) Kenics mixer (b) mixer: $C=1.25$ (c)mixer: $C=1.5$ and $(d)$ mixer: $C=2 ; B o x e d$ values $=$ average values.

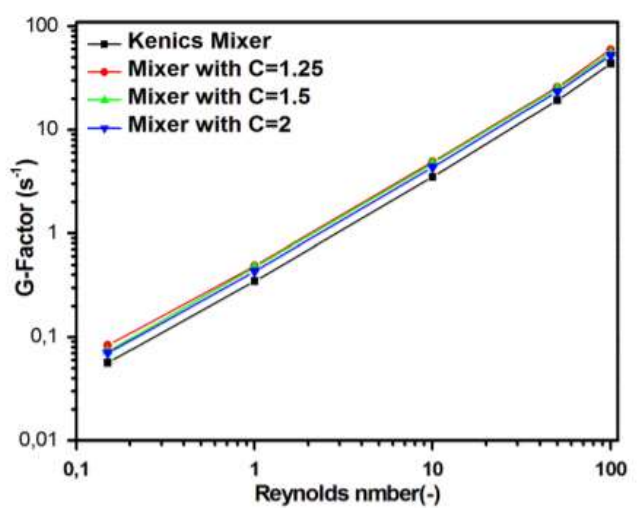

Fig. 10. G-factor vs. Reynolds number for different mixer Geometries.

efficiency in different mixing devices, particularly in various types of static mixers in dosing applications and water treatment Jones et al.(2002). Despite the widespread use of the G-factor with its associated limitations, it remains entrenched in the engineering literature as a valuable evaluation of mixing efficiency due to easiness to be numerically predicted. Consequently, in the present study, the Gfactor was adopted as an assessing criterion to quantify the performance of different mixers. The Gfactor is calculated based on the mixer volume, Reynolds number, and pressure drop that occurs in the given mixer as follows:

$G=\frac{1}{2} \sqrt{\frac{\pi D}{V_{m}}} \sqrt{\left.\frac{\operatorname{Re}(\Delta P)}{\rho}\right)}$

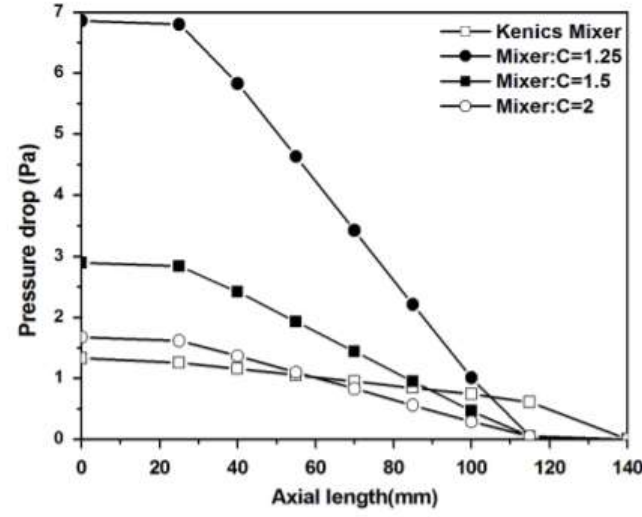

Fig. 11. Variation of pressure drop along the axial length for all mixer geometries at $\mathbf{R e}=100$.

Where: $V_{m}$ is the volume of the mixer, Fig. 10 shows the Logarithmic evolution of the G-factor as a function of the Reynolds number. It can be seen that the G-factor is proportional to the logarithm of the Reynolds number for all mixers. Consequently, the performance of the static mixer is improved with the increase of Reynolds number Re. For all values of $\mathrm{Re}$, the static mixer with $\mathrm{C}=1.25$ has the highest $\mathrm{G}-$ factor, followed by $\mathrm{C}=1.5, \mathrm{C}=2$, then the standard Kenics mixer. Incorporating secondary helical mixers in the mixing channel generates extra pressure drop because of the additional volume of helical mixer elements. The variation of pressure drop along the axial length of all mixers is elucidated in Fig. 11. It is remarked that the lower is the diameter aspect ratio $\mathrm{C}$, the higher is the pressure 
drop. The pressure drop in the mixers with $\mathrm{C}=1.5$ and $\mathrm{C}=2$ is less than the mixer: $\mathrm{C}=1.25$. Consequently, the dispersive mixing is enhanced at the expense of the pressure drop, where the best configuration based on low pressure drop and good dispersive mixing criteria is the mixer with aspect ratio $\mathrm{C}=2$.

\subsection{Mixture homogeneity and efficiency of mixing}

The intensity of segregation (IOS) method is employed as a criterion to evaluate the mixing quality of a mixture. Danckwerts (1952) originally suggested this method, and it characterizes the mixing process qualitatively. It measures the integrity and closeness of fluids concentration at a given cross-section to the mean concentration in the same sectional area in the mixer (i.e., the concentration variance of the liquid $\mathrm{A}$ and the liquid B "tracer" in the cells belongs to the same section). It has a value of 1 when the liquids are entirely separated. In contrast, the value of 0 delineates the complete mixing of liquids. The IOS is formulated as follows:

$$
I O S=\frac{\sigma_{h}^{2}}{\bar{h}(1-\bar{h})}
$$

Where $h$ the concentration at each is measured point, $\bar{h}$ is the average concentration of the sample at a given cross section, and $\sigma_{h}$ is the standard deviation of the concentration of the sampling points at a particular axial position. In the present work, $95 \%$ homogeneity is thought to be reached when the IOS value is 0.05 .

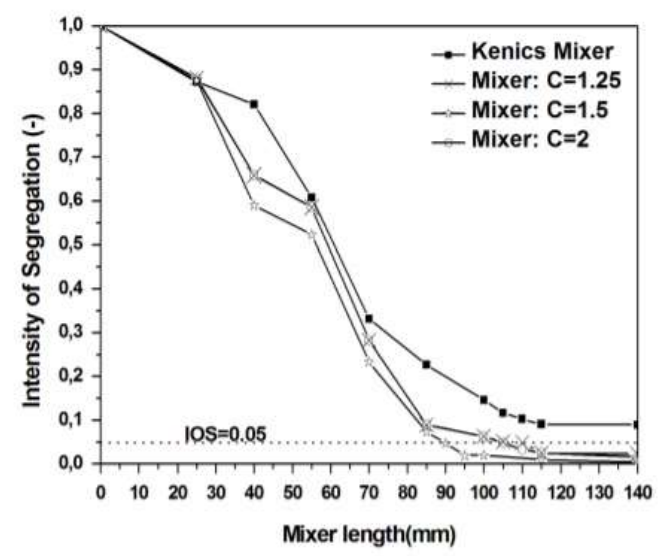

Fig. 12. Variation of intensity of segregation (IOS) for different mixer geometries along the flowdirection at $\mathbf{R e}=\mathbf{5 0}$.

Figure 12 shows the variation of Intensity of segregation (IOS) for different mixers with various diameter aspect ratios $\mathrm{C}$ at $\mathrm{Re}=50$. As noticed, the IOS is scarcely the same at the leading edge of the first mixer element for all mixer geometries. It decreases as the liquids pass through the overlapping mixer elements downstream due to the continuous blending of liquids streams. The secondary overlapped mixer deflects the flow direction and creates chaotic advection, enhancing the distributive mixing in the radial direction and overall mixing quality. The higher mixing efficiency in short mixing length is achieved with mixer: $\mathrm{C}=1.5$ at IOS and length values 0.0475 and $90 \mathrm{~mm}$, respectively $(95 \%$ uniformity reached in the first half-length of the 5th mixer element). The mixer: $\mathrm{C}=2$ reached IOS $=0.0487$ at length $105 \mathrm{~mm}(95 \%$ uniformity reached in first half-length of the 6th mixer element). Then the Mixer: $\mathrm{C}=1.25$ at a length of $110 \mathrm{~mm}$ with IOS $=0.0485$ ( $95 \%$ uniformity reached in second half-length of the 6th mixer element). The lower mixing efficiency of the modified mixers is obtained with a mixer of small diameter aspect ratio $\mathrm{C}$ (in our case $\mathrm{C}=1.25$ ) resulting from segregated regions created between the blades and the mixer wall because of higher axial velocity and high pressure, add to the formation of vortex cores with a rotational motion that deter the mass transfer of cores with the rest of the flow. In contrast, for the Kenics mixer, the required $95 \%$ homogeneity has not been reached even at the outlet $(\mathrm{L}=140 \mathrm{~mm})$ where IOS has only reached the value of 0.09 . Significantly, incorporating the overlapping helical mixers considerably increases the irregularities and surface contact of species. As the flow is laminar, the inertial forces are smaller to overcome the frictional forces and resistance due to blade geometry, because of the pressure decrease which is conversely proportional to the contact area. The blades block the fluid motion and force the species to have long journey, and sufficient time. There is a linear relation between time and diffusivity, hence slow motion promotes inter-material diffusion. The mixing is then ensured by molecular diffusion and the chaotic advection.

Figure 13 represents the mass fraction distribution of the tracer at the leading edge of the first mixer element and all the trailing edge of the mixer elements at $\mathrm{Re}=100$. The liquids flow side to side at the inlets and split into several streams for all mixers as the flow moves through the mixer elements. As can be seen, the two liquids are rapidly get combined just after the first mixing element, mainly for the configurations of the overlapping mixer. Whereas, for standard Kenics mixer, species are intermingled downstream until the third mixer element. Furthermore, the aspect ratio $\mathrm{C}$ has a strong effect on the mixing length, where the short mixing length is obtained as stated above with a mixer of $\mathrm{C}=1.5$ with $95 \%$ uniformity downstream at the 5th mixer element, which explains the disturbance provided by the edges of the overlapped blades, and ultimately improvement of mixing performance.

The value 0.05 of IOS indicates the complete uniformity of liquids. One should consider not only this value for IOS to decide to which extent is the efficiency of the static mixer. The mixer efficiency stands for the mean of reaching the mixing objective, where the same quality of mixing can be achieved by an efficient and inefficient static mixer design. The selection of an efficient static mixer is characterized by good mixedness, short mixing length, and low pressure drop.

Figure 14 illustrates the comparison of mixer efficiency of all mixer geometries with the same 


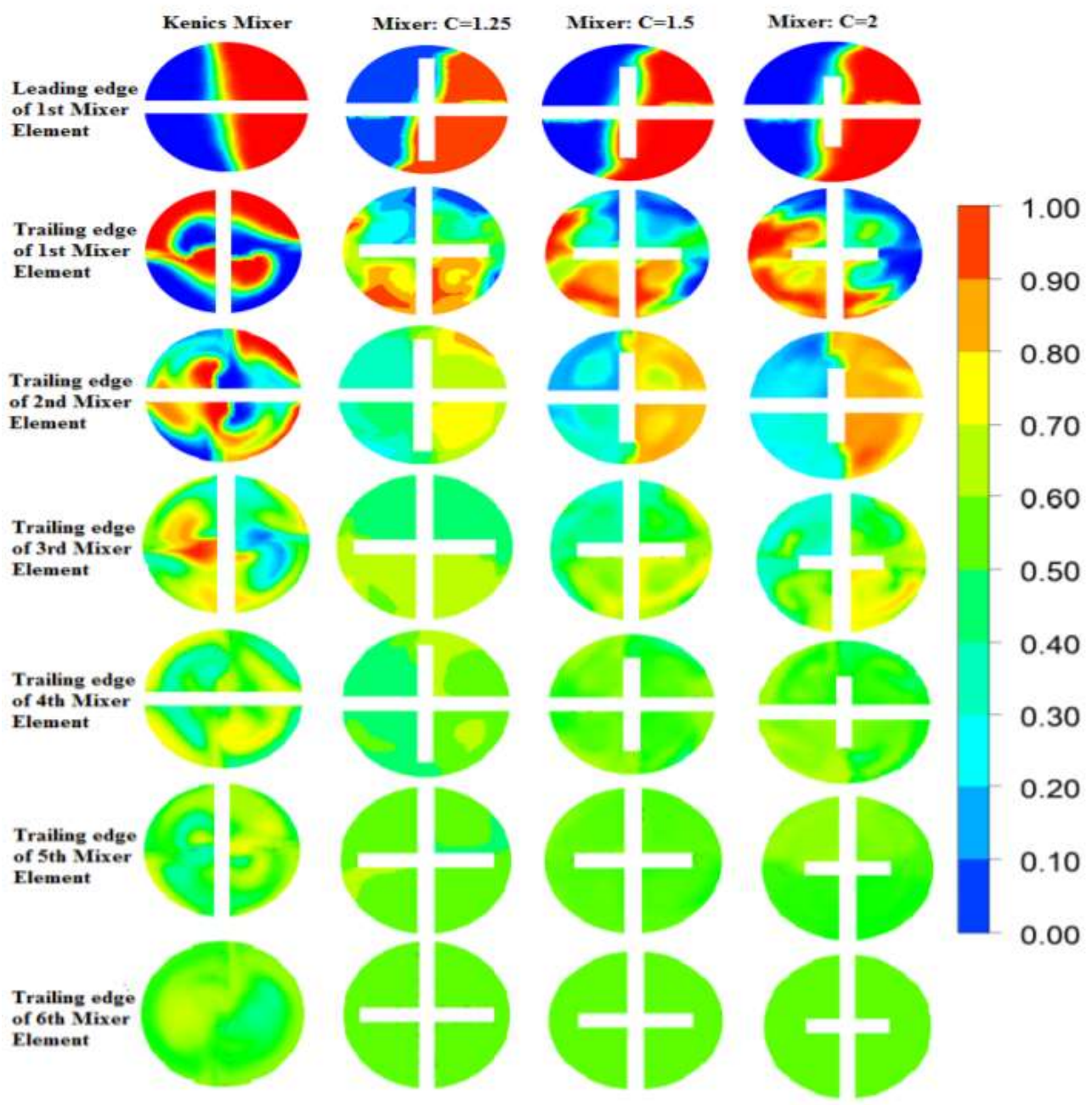

Fig. 13. Mass fraction distribution at various cross sections in different mixers: rows represent different mixers and columns represent cross sectional locations.

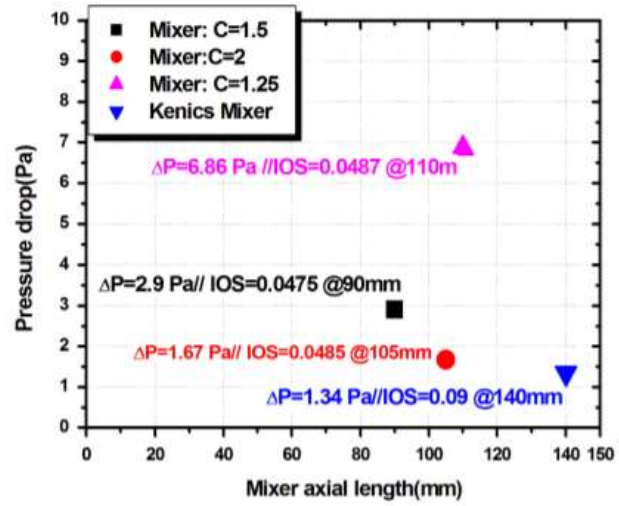

Fig. 14. Variation of mixer efficiency with length and pressure drop for $\mathrm{IOS}<0.05$. for $\mathrm{Re}=100$.

process conditions as a function of pressure drop, mixing length, and IOS less than 0.05 at Reynolds number $\mathrm{Re}=100$. It is clearly shown that the mixer: $\mathrm{C}=1.5$ is the most efficient static mixer in terms of short mixing length. It shows faster mixing at a length of $90 \mathrm{~mm}$ compared to other mixer geometries. Nevertheless, it is not the most efficient when the low pressure drop is prioritized over fast mixing. On the other hand, the Mixer: $\mathrm{C}=2$ exhibits a lower pressure drop with $73 \%$ less than the Mixer: $\mathrm{C}=1.5$ and $24 \%$ greater than the Kenics mixer, hence less energy consumption. Add to the short mixing length at $105 \mathrm{~mm}$ with an overall mixing homogeneity of $95.15 \%$. The mixers with $\mathrm{C}=1.25$ and the standard Kenics mixer are inefficient in both fast mixing and low pressure drop criteria.

\section{ConCLUSION}

The quality of mixing, pressure drop, extensional efficiency, and G-factor are essential measures to characterize the flow pattern and mixing performance in static mixers. These characteristic measurements are used to investigate the mixing performance in the Kenics mixer and new modified overlapping mixer geometries for a range of Reynolds numbers from 0.15 to 100 . The effect of incorporating secondary overlapping mixer elements in the main helical mixer with the opposite twist 
directions (RL-LR) on the mixing performance was also investigated. Furthermore, the effect of diameter aspect ratio $\mathrm{C}$ as the ratio of main mixer diameter $\mathrm{D}$ to diameter $\mathrm{d}$ of overlapping mixer was also examined.

The simulation results proved that incorporating a secondary overlapping mixer creates the disturbance, generates chaotic advection, and increases the contact area between the liquids, add to the reduction of molecular diffusion. Therefore, improvement in mixing performance can be achieved by new modified mixer geometries compared to Kenics mixer.

For the modified mixer geometries, the diameter of the overlapped mixer has a significant effect on the pressure drop within the mixer; the pressure drop increases when the $\mathrm{C}$ decreases. The results showed the same trend for the extensional efficiency and Gfactor; they increased by decreasing the aspect ratio C. In addition, the mixing quality is quantified in terms of Intensity Of Segregation (IOS) was examined for appropriate selection of efficient mixer for fast and short mixing length and/or low pressure drop. The optimal mixer is the mixer: $\mathrm{C}=2$ with less required power with a satisfactory tradeoff of a slightly high mixing length.

In light of findings in this paper, there is a concern about mixing these mixers with realistic conditions and their reliability for complex fluids. Future studies will evaluate the efficiency of these mixers for the mixing of immiscible and non-Newtonian fluids and transient, transitional, and turbulent flow regimes. Also, experimental investigations are indispensable to validate the findings in more realistic conditions.

\section{ACKNOWLEDGMENTS}

The authors are grateful to all cotributors for their technical support.

\section{REFERENCES}

Asprion, N., B. Rumpf and A. Gritsch (2011). Work flow in process development for energy efficient processes. Applied Thermal Engineering 31(13), 2067-2072.

Bakker, A., R. LaRoche and E. Marshall (2000). Laminar flow in static mixers with helical elements. The online CFM book 546.

Byrde, O. and M. Sawley (1999). Optimization of a kenics static mixer for non-creeping flow conditions. Chemical Engineering Journal 72(2), 163-169.

Camp, T. R. (1943). Velocity gradients and internal work in fluid motion. Journal of the Boston Society of Civil Engineer 30, 219-230.

Caserta, S., V. Preziosi, A. Pommella and S. Guido (2013). Non-newtonian liquid liquid fluids in kenics static mixers. Chemical Engineering Transactions 32, 1477-1482.
Danckwerts, P. V. (1952). The definition and measurement of some characteristics of mixtures. Applied Scientific Research 3(4), 279-296.

Galaktionov, O., P. Anderson, G. Peters and H. Meijer (2003). Analysis and optimization of kenics static mixers. International Polymer Processing 18(2), 138-150.

Ghanem, A., T. Lemenand, D. D. Valle and H. Peerhossaini (2014). Static mixers:Mechanisms, applications, and characterization methodsa review. Chemical Engineering Research and Design 92(2), 205228.

Grace, C. (1971). Static mixing and heat transfer. Chem. Chemical and Process Engineering 52, 57-59.

Haddadi, M., S. Hosseini, D. Rashtchian and M. Olazar (2020). Comparative analysis of different static mixers performance by cfd technique: An innovative mixer. Chinese Journal of Chemical Engineering 28(3), 672684.

Hobbs, D. and F. Muzzio (1997). The kenics static mixer: A three-dimensional chaotic flow. Chemical Engineering Journal 67(3), 153 166.

Hobbs, D. and F. Muzzio (1998). Optimization of a static mixer using dynamical systems techniques. Chemical Engineering Sciencel 53(18), 3199-3213.

Jovanovi, A., M. Pezo, L. Pezo and L. Levi (2014). Dem/cfd analysis of granular flow instatic mixers. Powder Technology 266, 240- 248.

Lisboa, P., J. Fernandes, P. Simes, J. Mota and E. Saatdjian (2010). Computational-fluid dynamics study of a kenics static mixer as a heat exchanger for supercritical carbon dioxide. The Journal of Supercritical Fluids 55(1), 107-115.

Mahammedi, A., H. Ameur and A. Ariss (2017). Numerical investigation of the performance of kenics static mixers for the agitation of shear thinning fluids. Journal of applied fluid . Journal of Applied Fluid Mechanics 10(3), 989-999.

Manas-Zloczower, I. (1994). Studies of mixing efficiency in batch and continuous mixers. Rubber Chemistry and Technology 67(3),504528.

Meng, H., M. Song, F. Wang and J. Wu (2015). Chaotic mixing characteristics in static mixers with different axial twistedtape inserts.The Canadian Journal of Chemical Engineering 93(10), 1849-1859.

Meng, H., M. Song, Y. Yu, X. Jiang, Z. Wang and J. $\mathrm{Wu}$ (2014). A numerical study of mixing performance of high-viscosity fluid in novel static mixers with multitwisted leaves. 
Industrial \& Engineering Chemistry Research 53(10), 4084-4095.

Meng, H., M. Song, Y. Yu, X. Jiang, Z. Wang and J. $\mathrm{Wu}$ (2016a). Enhancement of laminar flow and mixing performance in a lightning static mixer. International Journal of Chemical Reactor Engineering 15(3).

Meng, H., G. Zhu, and Z. W. and J. Wu (2016b). The effect of symmetrical perforated holes on the turbulent heat transfer in the static mixer with modified kenics segments. International Journal of Heat and Mass Transfer 99, 647659.

Heniche, M., P. A. Tanguy, M. F. Reeder and J. Fasano (2005). Numerical investigation of blade shape in static mixing. AIChE Journal 51(1), 44-58.

Murasiewicz, H. and Z. Jaworski (2009). Transient cfd simulations of turbulent liquid liquid flow in a kenics static mixer. Polish Journal of Chemical Technolog 11(2), 36-40.

Rafiee, M., M. Simmons, A. Ingram and E. Stitt (2013). Development of positron emission particle tracking for studying laminar mixing in kenics static mixer. Chemical Engineering Research and Design 91(11), 2106-2113.

Rahmani, R., T. Keith and A. Ayasoufi (2008). Numerical simulation of turbulent flow in an industrial helical static mixer. International Journal of Numerical Methods for Heat and Fluid Flow 18(6), 675-696.

Ramsay, J., M. Simmons, A. Ingram and E. Stitt (2016). Mixing performance of viscoelastic fluids in a kenics $\mathrm{km}$ in-line static mixer. Chemical Engineering Research and Design 115, 310-324.

Rauline, D., J. L. Blevec, J. Bousquet and P. Tanguy (2000). A comparative assessment of the performance of the kenics and smx static mixers. Chemical Engineering Research and Design 78(3), 389-396.

Rauline, D., P. A. Tanguy, J. L. Blvec and J. Bousquet (1998). Numerical investigation of the performance of several static mixers. The Canadian Journal of Chemical Engineering 76(3), 527-535.

Regner, M., K. stergren and C. Trgrdh (2006). Effects of geometry and flow rate on secondary flow and the mixing process in static mixersa numerical study. Chemical Engineering Science 61(18), 6133-6141.
Saatdjian, E., A. Rodrigo and J. Mota (2012). On chaotic advection in a static mixer. Chemical Engineering Journal 187(1), 289-298.

Jegatheeswaran, S., F. Ein-Mozaffari and J. Wu (2017). Efficient mixing of yield pseudoplastic fluids at low reynolds numbers in the chaotic smx static mixer. Chemical Engineering Journal 317, 215-231.

Jones, S., C. F. Sotiropoulos and A. Amirtharajah (2002). Numerical modeling of helical static mixers for water treatment. Journal of Environmental Engineering 128(5), 413-440.

Soman, S. and C. Madhuranthakam (2017). Effects of internal geometry modifications on the dispersive and distributive mixing in static mixers. Chemical Engineering and Processing: Process Intensification 122, 31-43.

Song, H. and S. Han (2005). A general correlation for pressure drop in a kenics static mixer. Chemical Engineering Science 60(21), 56965704.

Stec, M. and P. Synowiec (2019). Study of fluid dynamic conditions in the selected static mixers part iiiresearch of mixture homogeneity. The Canadian Journal of Chemical Engineering 97(4), 995-1007.

Thakur, R., C. Vial, K. Nigam, E. Nauman and G. Djelveh (2003). Static mixers in the process industriesa review. Chemical Engineering Research and Design 81(7), 787-826.

Tian, S. and M. Barigou (2015). An improved vibration technique for enhancing temperature uniformity and heat transfer in viscous fluid flow. Chemical Engineering Science 123, 609619.

Vilar, V., A. Diez, F. Moreira, B. Marinho, J. Espnola, R. Martins and R. Boaventura (2017). Intensification of heterogeneous photocatalysis by applying a kenics static mixer as catalyst support. 3rd Iberoamerican Conference on Advanced Oxidation Technologies (III Cipoa) and 2nd Colombian Conference on Advanced Oxidation Processes (II Ccpaox).

Wilkinson, W. and M. Cliff (1977). An investigation into the performance of a static in line mixer. Second European Conference on Mixing, BHRA Fluid Engineering, Cambridge 1, 15-25.

Zhang, L., J. Dong, B. Jiang, Y. Sun, F. Zhang and L. Hao (2015). A study of mixing performance of polyacrylamide solutions in a new-type static mixer combination. Chemical Engineering and Processing: Process Intensification 88, 19-28. 